\title{
Article \\ Non-Inferiority Field Study Comparing the Administrations by Conventional Needle-Syringe and Needle-Free Injectors of a Trivalent Vaccine Containing Porcine Circovirus Types 2a/2b and Mycoplasma hyopneumoniae
}

\author{
Hyejean Cho, Yongjun Ahn, Taehwan Oh D, Jeongmin Suh and Chanhee Chae *
}

check for updates

Citation: Cho, H.; Ahn, Y.; Oh, T.; Suh, J.; Chae, C. Non-Inferiority Field Study Comparing the Administrations by Conventional Needle-Syringe and Needle-Free Injectors of a Trivalent Vaccine Containing Porcine Circovirus Types $2 \mathrm{a} / 2 \mathrm{~b}$ and Mycoplasma hyopneumoniae. Vaccines 2022, 10, 358. https:// doi.org/10.3390/vaccines10030358

Academic Editor: François Meurens

Received: 12 January 2022

Accepted: 21 February 2022

Published: 24 February 2022

Publisher's Note: MDPI stays neutral with regard to jurisdictional claims in published maps and institutional affiliations.

Copyright: (c) 2022 by the authors. Licensee MDPI, Basel, Switzerland. This article is an open access article distributed under the terms and conditions of the Creative Commons Attribution (CC BY) license (https:// creativecommons.org/licenses/by/ $4.0 /)$.

\author{
Department of Veterinary Pathology, College of Veterinary Medicine, Seoul National University, Gwanak-ro 1, \\ Gwanak-gu, Seoul 08826, Korea; hcho21@snu.ac.kr (H.C.); ayj3ca@gmail.com (Y.A.); ohth93@gmail.com (T.O.); \\ tobin1210@snu.ac.kr (J.S.) \\ * Correspondence: swine@snu.ac.kr
}

\begin{abstract}
The objective of this study was to assess the clinical, immunological, microbiological, and pathological evaluation of trivalent vaccine containing porcine circovirus types $2 a / b(P C V 2 a / b)$ and Mycoplasma hyopneumoniae given by two different needle-free injection devices compared with conventional needle-syringe injection in a herd with subclinical PCV2d infection and enzootic pneumonia. A total of 240 21-day-old pigs, which weighed between 5 to $6 \mathrm{~kg}$, were randomly divided into four groups ( 60 pigs per group, $30=$ male and $30=$ female per group). Injection site reactions in the pigs were minimal for the two needle-free injection devices and needle-syringe injection. Trivalent vaccination of pigs with two needle-free injection devices was not inferior to conventional needle-syringe injection for growth performance. Trivalent vaccination of pigs with two different needle-free injection devices reduced levels of PCV2d loads in serum and M. hyopneumoniae loads in the larynx equally compared to the conventional needle-syringe injection. The amount of PCV2d load in serum from the needle-free Pulse FX injection device at 49 days post vaccination showed non-inferiority to conventional needle-syringe injection. The immune response against PCV2 and M. hyopneumoniae to trivalent vaccine given with the needle-free Pulse FX injection device was non-inferior to conventional needle-syringe injection. The pigs from the two needle-free injection device and conventional needle-syringe injection had significantly $(p<0.05)$ lower macroscopic and microscopic lung lesion scores, and microscopic lymphoid lesions than from unvaccinated. The results of this study demonstrated that vaccination of trivalent vaccine by the two needle-free Pulse injection devices used in the study was non-inferior to that by conventional needle-syringe injection for growth performance, immune response against PCV2 and M. hyopneumoniae, and reduction of PCV2 viremia.
\end{abstract}

Keywords: Mycoplasma hyopneumoniae; needle-syringe; needle-free injector; porcine circovirus type 2; vaccine

\section{Introduction}

Porcine circovirus type 2 (PCV2) and Mycoplasma hyopneumoniae are two major economically important pathogens in global pork industry. PCV2 is the principal causative agent of porcine circovirus-associated disease (PCVAD), which was originally described as postweaning multisystemic wasting syndrome [1,2]. Subclinical PCV2 infection is currently the most common form of PCVAD [3]. Additional disease manifestations such as porcine respiratory disease complex (PRDC), and reproductive failure have been found in all ages of swine [1]. M. hyopneumoniae lacks a cell wall and is one of the smallest bacteria found in nature. As a primary contributor to PRDC, M. hyopneumoniae is the etiological agent of enzootic pneumonia [4]. Although it is not a new pathogen, for these reasons, it is one of the most economically devastating pathogens to the swine industry. 
PCV2 and M. hyopneumoniae play an important role in PRDC [4,5]. Control of these two pathogens is heavily dependent on vaccination. Immunization via needle-syringe injection has been in practice since the origin of vaccinology in veterinary medicine, but such conventional vaccination presents few challenges, as it is time-consuming for swine workers and stressful for pigs, as fear and pain indicators at the time of injection are increased [6]. Needle-free injection devices offer advantages over conventional needlesyringe injection, such as increased safety of swine workers, less pain and stress of pigs, avoidance of broken needles, and less risk of iatrogenic pathogen transmission between pigs caused by reusing needles [6-10]. The elimination of broken needles and associated trim at the process is the most important of these elements in pork quality assurance. Consequently, interest in the use of a needle-free device system has increased over the last three years in the Asian and global pig industry.

A new trivalent PCV2a/b and M. hyopneumoniae vaccine (Fostera ${ }^{\circledR}$ Gold PCV MH /CircoMax ${ }^{\circledR}$ Myco, Zoetis, Parsippany, NJ, USA) has recently been introduced into the market [11]. The PCV2b antigen in this trivalent vaccine is genetically similar to PCV2d, which was formerly referred to as 'mutant PCV2b'. Currently, PCV2d is the most prevalent field genotype spread worldwide [12-14]. Despite the advantages of needle-free injection devices over conventional needle-syringe injection, clinical trials on needle-free injection devices have not yet been reported for this new vaccine. The objective of this study was to compare the effectiveness of two different needle-free injection devices with conventional needle-syringe used to administer a trivalent $\mathrm{PCV} 2 \mathrm{a} / \mathrm{b}$ and $\mathrm{M}$. hyopneumoniae vaccine in a pig herd suffered from subclinical PCV2d infection and enzootic pneumonia, in relation to growth performance, induction of PCV2 and M. hyopneumoniae antibodies, reduction of PCV2d viremia and mycoplasmal laryngeal shedding, and the reduction of pathological lesions under field conditions.

\section{Materials and Methods}

\subsection{Farm}

The clinical field trial was conducted on a 1200-Large White $\times$ Landrace cross bred sow farrow-to-finish swine farm with an all-in-all-out production system. Farm selection was based on clinical history of subclinical PCV2 infection and enzootic pneumonia. The chosen farm status of porcine reproductive and respiratory syndrome (PRRS) was seropositive but absence of clinical signs of PRRS in breeding-herd population. A detailed clinical history of the farm was described elsewhere [15].

\subsection{Experimental Design}

A total of 240 21-day-old pigs, weighing 5 to $6 \mathrm{~kg}$ each, were randomly divided into four groups ( 60 pigs per group, $30=$ male and $30=$ female per group) using the random number generator function (Excel, Microsoft Corporation, Redmond, Washington, DC, USA). The pigs in the VacS, VacPulse, and VacEPIG groups were intramuscularly vaccinated with a $2.0 \mathrm{~mL}$ dose of Fostera Gold PCV MH (Serial no: 413369A, Expiration date: 03-Feb-2022, Zoetis, Parsippany, NJ, USA) by either conventional needle-syringe injection (VacS group), needle-free Pulse FX injection device (Serial no. FX-200-0111, Pulse NeedleFree System Inc., Lenexa, KS, USA) (VacPulse group), or by needle-free EPIG injection device (Serial no. 1553569, Henke-Sass Wolf GmbH, Tuttlingen, Germany) (VacEPIG group), respectively, at 21 days of age. Conventional needle-syringe-based vaccination was administered intramuscularly with a pistol-grip syringe fitted with a $10 \mathrm{~mm}$, 22-gauge needle (KOVAX-SYRINGE, Korean Vaccine, Seoul, Republic of Korea). Compressed $\mathrm{CO}_{2}$ was used as the power source for the needle-free Pulse FX injection device. Using guidelines from Pulse Needle Free Systems Inc, the device was set at a pressure of 85 to 95 pounders per square inch (PSI) on 21 days old pigs to deliver the intramuscular injection of $2.0 \mathrm{~mL}$ per pig. The needle-free EPIG injection device was set at the default setting of a $2.0 \mathrm{~mL}$ dosage. The pigs in the UnVac group were intramuscularly vaccinated 
with a $2.0 \mathrm{~mL}$ dose of phosphate buffered saline (PBS, $0.01 \mathrm{M}, \mathrm{pH} 7.4$ ) by conventional needle syringe at 21 days of age (Table 1 ).

Table 1. Field experimental design.

\begin{tabular}{|c|c|c|c|c|}
\hline Groups & No. of Pigs & $\begin{array}{c}\text { Injection } \\
\text { Instrument }\end{array}$ & Dosage & Age (Days) \\
\hline VacS & 60 & Syringe & One $(2.0 \mathrm{~mL})$ & 21 \\
\hline VacPulse & 60 & $\begin{array}{c}\text { Needle-free } \\
\text { Pulse FX device }\end{array}$ & One $(2.0 \mathrm{~mL})$ & 21 \\
\hline VacEPIG & 60 & $\begin{array}{l}\text { Needle-free } \\
\text { EPIG device }\end{array}$ & One $(2.0 \mathrm{~mL})$ & 21 \\
\hline UnVac & 60 & Syringe & One $(2.0 \mathrm{~mL})$ & 21 \\
\hline
\end{tabular}

Pigs were comingled with treatment groups and randomly assigned into 12 pens within the same building. Each pen contained 20 pigs with a same proportion of each treatment per pen (five pigs per group). Pens were identical in design and equipment, which included free access to a feed and water trough. Blood and laryngeal swabs were collected at 0 (21 days of age), 28 (49 days of age), 49 (70 days of age), and 91 (112 days of age) days post-vaccination ( $\mathrm{dpv}$ ). All the methods were approved by the Seoul National University Institutional Animal Care and Use, and Ethics Committee (SNU-210518-3).

\subsection{Post-Vaccination Skin Reaction}

Presence of vaccine residue at the skin surface was recorded immediately following vaccination. Pigs were visually scored for the presence of post-vaccination skin reactions at $0,1,4,7,14$, and $21 \mathrm{dpv}$. Any raised surface observed at the injection site was considered a skin reaction occurring as a result of vaccination.

\subsection{Clinical Observations}

The pigs were monitored daily for abnormal clinical signs and scored weekly using scores ranging from 0 (normal) to 6 (severe dyspnea and abdominal breathing) [16]. Observers were blinded to vaccination and type of vaccine status. Mortality rate was calculated as the number of pigs that died divided by the number of pigs initially assigned to that group. Pigs that died or were culled throughout the study were necropsied.

\subsection{Average Daily Weight Gain}

The live weight of each pig was measured at 0 ( 21 days of age), 49 (70 days of age), and 154 (175 days of age, or slaughter day) days post-vaccination. The average daily weight gain (ADWG; grams/pig/day) was analyzed over two time periods: (i) between 21 and 70, and (ii) between 70 and 175. ADWG during the different production stages was calculated as the difference between the starting and final weight divided by the duration of the stage. Data of body weight for dead or removed pigs were included in the calculation.

\subsection{Serology}

The serum samples were tested using the commercially available PCV2 (INgezim CIRCO IgG, Ingenasa) and M. hyopneumoniae (M. hyo. Ab test, IDEXX Laboratories Inc., Westbrook, ME, USA) ELISA kits. Samples were considered positive for PCV2 antibodies if the optical density (OD) was $>0.3$ and for $M$. hyopneumoniae antibodies if the sample-topositive $(\mathrm{S} / \mathrm{P})$ ratio was $\geq 0.4$, according to the manufacturer's instructions.

\subsection{Quantification of PCV2d DNA in Serum}

DNA was extracted from serum samples using the commercial kit (QIAamp DNA Mini Kit, QIAGEN, Valencia, CA, USA) to quantify PCV2d genomic DNA copy numbers by real-time PCR [2]. 


\subsection{Quantification of M. hyopneumoniae DNA in Larynx}

DNA was extracted from laryngeal swabs using the commercial kit (QIAamp DNA Mini Kit, QIAGEN) to quantify M. hyopneumoniae DNA copy numbers by real-time PCR [17].

\subsection{Pathology}

The severity of macroscopic lung lesions was scored to estimate the percentage of the lung affected by pneumonia. The scoring was done by two pathologists (Chae and one graduate student) at the Seoul National University (Seoul, Republic of Korea). For the entire lung, 100 points were assigned as follows; 10 points each to the right cranial lobe, right middle lobe, left cranial lobe, and left middle lobe, 27.5 points each to the right caudal lobe and left caudal lobe, and 5 points to the accessory lobe [16]. Two blinded veterinary pathologists then examined the collected lung and lymphoid tissue sections and scored the severity of peribronchiolar lymphoid tissue hyperplasia by mycoplasmal pneumonia lesions (0 to 6) [18]. Lymphoid lesion severity was scored (0 to 5) based on lymphoid depletion and granulomatous inflammation [19].

\subsection{Statistical Analysis}

Prior to statistical analysis, real-time PCR data were transformed to $\log _{10}$ values. The Shapiro-Wilk test was used to test the collected data for a normal distribution. Results were reported in $p$-Value, where a value of $p<0.05$ was considered significant.

ADWG, PCV2 and M. hyopneumoniae ELISA, and real-time PCR PCV2 and M. hyopneumoniae DNA quantification data were analyzed using a generalized linear mixed model for repeated measures with the fixed effects which includes treatment, time point, and their interaction. As the random effects, pen and animal are included. Each time point estimation has the least squares means (or geometric means for PCR data and ELISA titer) and the relative 95\% confidence intervals (CI) limits. For ADWG, PCV2 and M. hyopneumoniae ELISA, and real-time PCR PCV2 and M. hyopneumoniae DNA quantification results, the non-inferiority trial was conducted to compare between needle-free injection (VacPulse and VacEPIG) groups within the reference group, the conventional needle-syringe injection (VacS) groups. At the time points, 49 (70 days of age) and 91 (112 days of age), the reference group (VacS) was at the $2.5 \%$ level for one-sided tests, and the least squares means (delta $10 \%)$.

Macroscopic lung lesions (scores between 0 and 100), microscopic lung (scores between 0 and 6) and microscopic lymphoid (scores between 0 and 5) lesions, and mortality were analyzed using a generalized linear mixed model for binomial data with the fixed effect of treatment. The model includes the random effect of pen. The optimal cutoff value for binary outcomes of macroscopic lung lesions, microscopic lung and microscopic lymphoid lesions were determined as $>20,>1$ and $>1$, respectively. The back transformed least squares means and their $95 \% \mathrm{CI}$ were provided. A treatment comparison was made between the needle-free injection (VacPulse and VacEPIG) groups and the reference (VacS) group when the treatment effect is significant in the model.

Clinical signs (normal $=0 /$ abnormal $1>0$ ) were analyzed using a generalized linear mixed model with repeated measurements for binomial data with the fixed effect of treatment, time point, and their interaction. The model includes the random effects of pen and animal. The back transformed least squares means and their 95\%CI were provided at each time point. The treatment comparison was made between the needle-free injection (VacPulse and VacEPIG) groups and the reference (VacS) group if any of treatment related effects were significant in the model.

\section{Results}

\subsection{Post-Vaccination Skin Reactions}

Substantial amounts of visible vaccine residue remained on the skin at the site of vaccination for both needle-free injection devices; $30 \%$, or 18/60 pigs from the VacPulse group and $20 \%$, or $12 / 60$ pigs from the VacEPIG group. No visible vaccine residues were 
not seen in pigs from the VacS group. The injection site reactions were minimal for all vaccine administration types, and injection site reactions that were identified were small swelling, ranging 2-3 in diameter, and self-resolved within 7 days after vaccination. Needlefree devices produced small scars in the injection site area in 4 out of 60 pigs from the VacPulse group and 5 out of 60 pigs from the VacEPIG group.

\subsection{Clinical Signs}

Respiratory sign scores were significantly lower $(p<0.05)$ in the vaccinated pigs (VacS, VacPulse, and VacEPIG groups) than those in unvaccinated pigs (UnVac group) at 28 to $98 \mathrm{dpv}$. Respiratory sign scores of needle-free injection device-vaccinated pigs in the VacPulse and VacEPIG groups were not statistically different from those of the needle-syringe injection-vaccinated pigs within the VacS group.

\subsection{Mortality}

One 82-day-old pig in the VacS group died of bronchopneumonia as diagnosed by isolation of Pasteurella multocida and Glaesserella parasuis in the lung. One 77-day-old pig in the VacPulse group died of bronchopneumonia as diagnosed with detection of M. hyopneumoniae by PCR and isolation of G. parasuis in the lung. Two pigs aged 67 and 72 days in the VacEPIG group died of bronchopneumonia as diagnosed by the detection of M. hyopneumoniae with PCR and isolation of Trueperella pyogenes in the lung. A total of five pigs died in the UnVac group; three pigs aged 59,62, and 93 days died of bronchopneumonia as diagnosis by detection of PCV2d and M. hyopneumoniae with PCR, and isolation of P. multocida in the lung; two pigs aged 71 and 82 days died of bronchopneumonia as diagnosis by detection of M. hyopneumoniae with PCR, and isolation of P. multocida in the lung.

\subsection{Average Daily Weight Gain}

Body weights were not significantly different at the beginning of study (21 days of age), but were statistical different at the end of the study (175 days of age) between the three vaccinated (VacS, VacPulse, and VacEPIG) and the unvaccinated (UnVac/UnCh) group. The ADWG of vaccinated pigs in the VacS, VacPulse, and VacEPIG groups was significantly higher $(p<0.05)$ than that of unvaccinated pigs in the UnVac group during the fattening period (70 to 175 days of age) and overall period (21 to 175 days). There was no statistical difference in ADWG between vaccinated groups (Table 2).

Table 2. Body weight and average daily weight gain (ADWG) data (mean \pm standard deviation) from vaccinated and unvaccinated groups.

\begin{tabular}{cccccc}
\hline & \multirow{2}{*}{$\begin{array}{c}\text { Age } \\
\text { (Days) }\end{array}$} & \multicolumn{3}{c}{ Groups } \\
\cline { 3 - 6 } & 21 & VacS & VacPulse & VacEPIG & UnVac \\
\hline Body weight & 175 & $105.65 \pm 2.46^{\mathrm{a}}$ & $105.32 \pm 2.24^{\mathrm{a}}$ & $104.93 \pm 1.99^{\mathrm{a}}$ & $99.30 \pm 2.68^{\mathrm{b}}$ \\
(Kg) & $21-70$ & $392.82 \pm 39.44$ & $388.71 \pm 31.96$ & $391.87 \pm 31.50$ & $376.50 \pm 33.16$ \\
ADWG & $70-175$ & $765.21 \pm 31.16^{\mathrm{a}}$ & $764.78 \pm 23.91^{\mathrm{a}}$ & $759.41 \pm 22.76^{\mathrm{a}}$ & $713.04 \pm 26.84^{\mathrm{b}}$ \\
$($ gram/pig/day) & $21-175$ & $647.03 \pm 15.32^{\mathrm{a}}$ & $645.14 \pm 15.04^{\mathrm{a}}$ & $642.55 \pm 13.12^{\mathrm{a}}$ & $606.36 \pm 17.86^{\mathrm{b}}$ \\
\hline
\end{tabular}

Different superscripts (a and $\mathrm{b}$ ) indicate significant $(p<0.05)$ difference among 4 groups.

For the ADWG, during fattening period (70 to 175 days) and overall period (21 to 175 days), the lower bound of the one-sided 95\%CI were greater than Delta (the 100\% margin of non-inferiority), indicating that the effectiveness of administration via both needle-free injection devices were non-inferior to effectiveness of injection via conventional needle-syringe (Figure 1). 


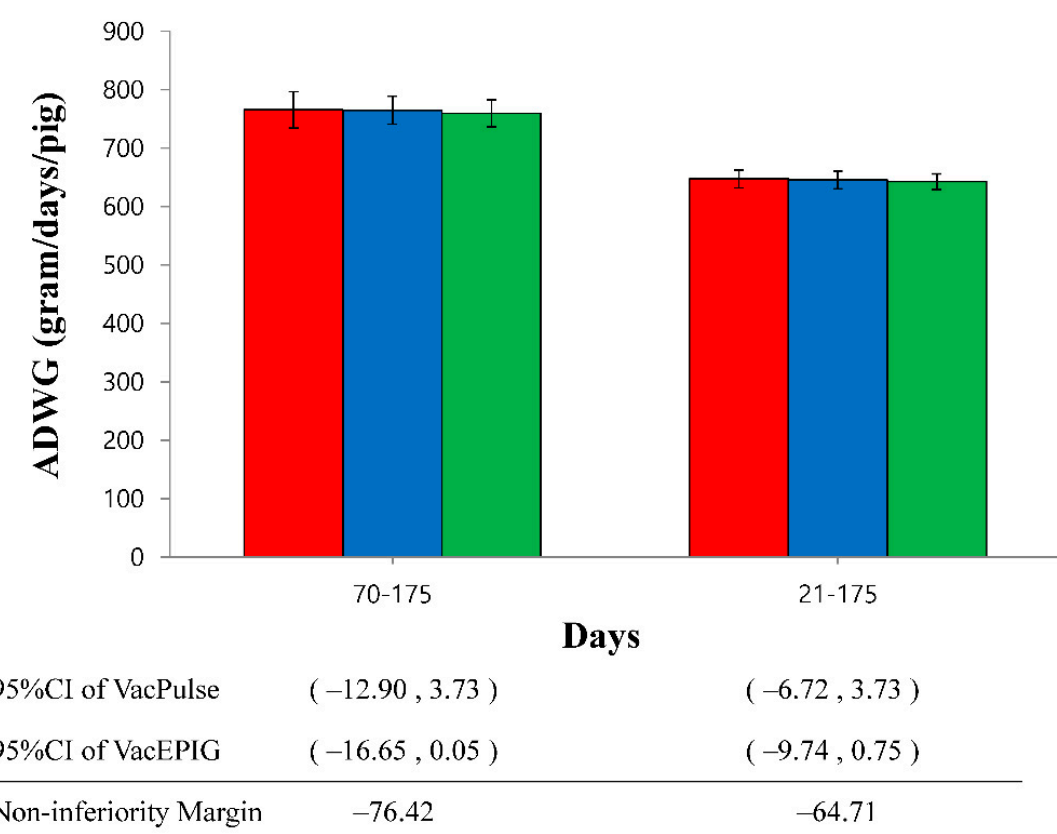

Figure 1. Non-inferiority analysis of average daily weight gain (ADWG) for 2 needle-free devices (VacPulse group •, and VacEPIG group, •) during fattening period (70 to 175 days) and overall period (21 to 175 days) as compared with conventional needle-syringe (VacS group, $\bullet$ ). The lower bound of the one-sided $95 \%$ confidence intervals (CI) was greater than the margin of non-inferiority, indicating that two needle-free devices were non-inferior to conventional needle-syringe.

\subsection{Quantification of PCV2d DNA in Serum}

The amount of PCV2d DNA measured in the serum from needle-free device-vaccinated pigs (VacPulse and VacEPIG groups) was significantly $(p<0.05)$ lower at $28 \mathrm{dpv}$ than that of unvaccinated pigs in the UnVac group. The amount of PCV2d DNA in serum of vaccinated pigs from the VacS, VacPulse, and VacEPIG groups was significantly $(p<0.05)$ lower at 49 and $91 \mathrm{dpv}$ than that measured for unvaccinated pigs in the UnVac group (Figure 2).

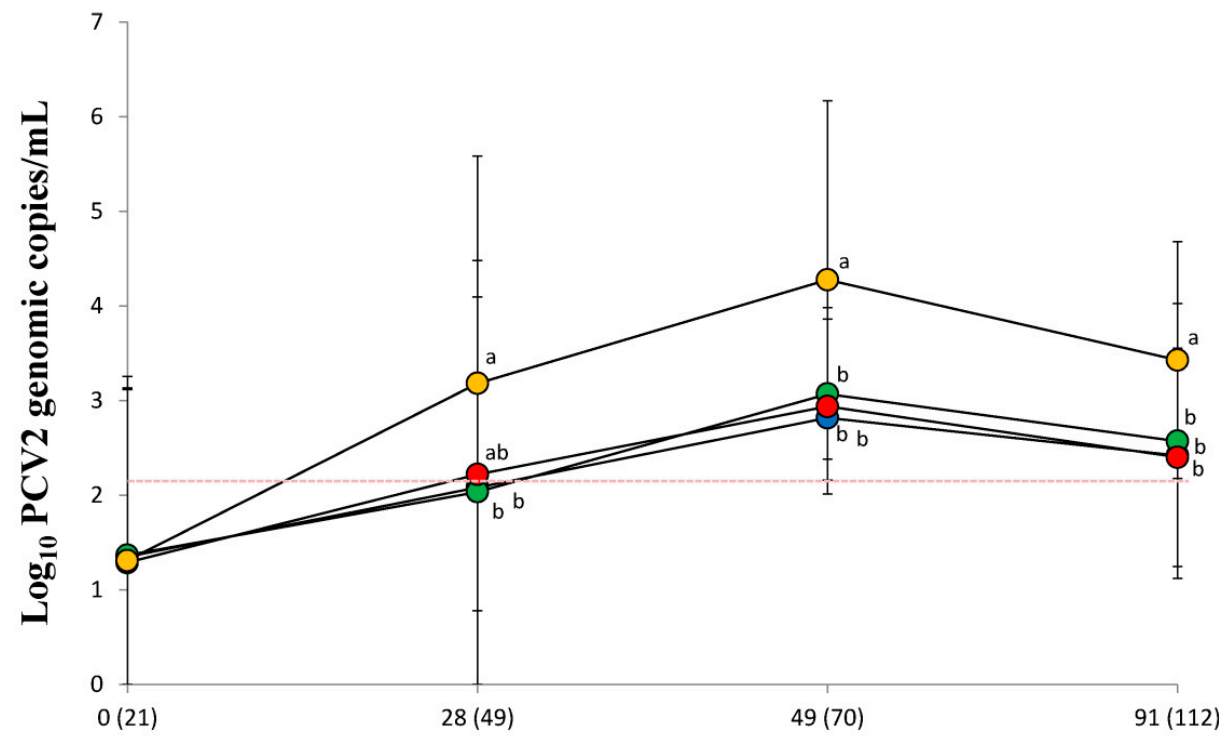

Days post-vaccination (age, days)

Figure 2. Mean values of the genomic copy number of PCV2d DNA in serum from VacS $(\bullet), \operatorname{VacPulse}$ $(\bullet)$, VacEPIG $(\bullet)$, and UnVac $(\bullet)$ groups. Variation is expressed as the standard deviation. The detection limit of the assay is $1.3 \times 10^{2}$ genomic copy numbers of PCV2d (red dotted line). Different superscripts $(a$ and $b)$ indicate significant $(p<0.05)$ different among 4 groups. 
The amount of PCV2d DNA in serum from the needle-free device (VacPulse) group at $49 \mathrm{dpv}$ showed non-inferiority to conventional needle-syringe injection (VacS group) (95\%CI -0.44 to 0.20 , non-inferiority margin 0.29 ). The upper limits of the one-sided $95 \%$ CI on the difference in the amount of PCV2d DNA in the serum from the VacPulse and VacEPIG groups at $91 \mathrm{dpv}(95 \% \mathrm{CI}$ for the VacPulse group -0.41 to 0.46 and the VacEPIG group -0.27 to 0.61 ) exceeded non-inferiority margin (0.24), indicating the outcome did not show non-inferiority (Figure 3).

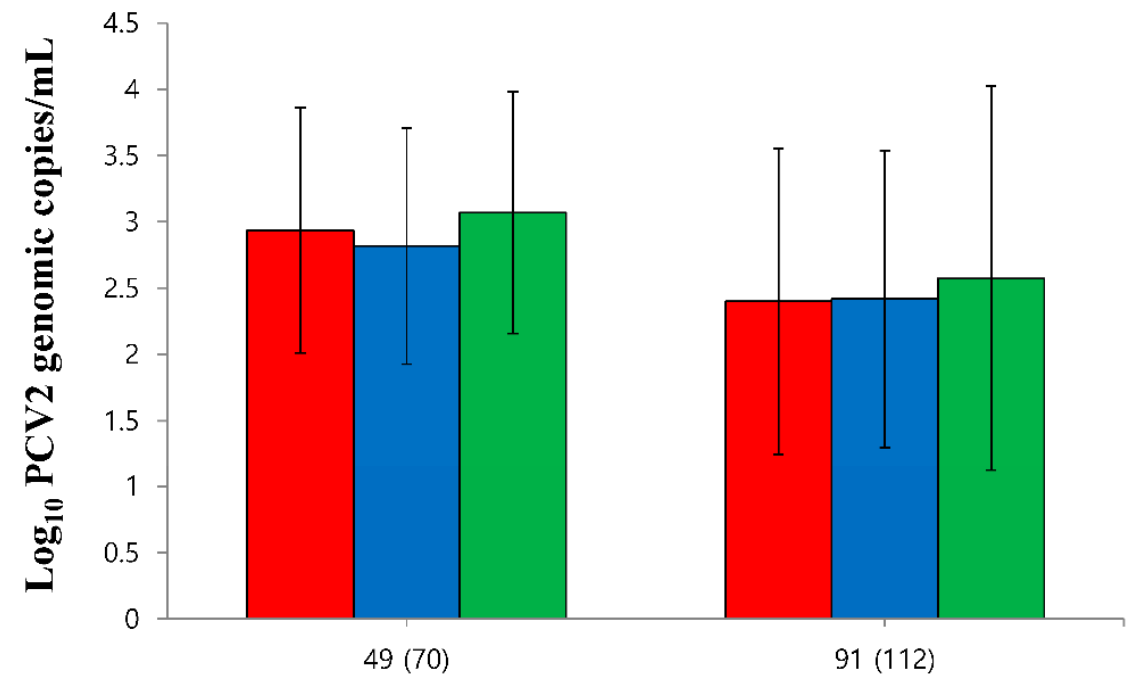

Days post-vaccination (age, days)

\begin{tabular}{lcc}
$95 \% \mathrm{CI}$ of VacPulse & $(-0.44,0.20)$ & $(-0.41,0.46)$ \\
$95 \% \mathrm{CI}$ of VacEPIG & $(-0.19,0.45)$ & $(-0.27,0.61)$ \\
\hline Non-inferiority Margin & 0.29 & 0.24
\end{tabular}

Figure 3. The upper bound of the $95 \%$ confidence intervals (CI) on the difference in PCV2d load in serum in the needle-free device (VacPulse, •) group at 49 days post-vaccination (dpv) showed non-inferiority to conventional needle-syringe (VacS, $\bullet$ group. The PCV2d loads in serum of needlefree device (VacEPIC, •) group at 49 and $91 \mathrm{dpv}$ did not show non-inferiority to conventional needle-syringe $(\mathrm{VacS}, \bullet)$ group.

\subsection{Quantification of M. hyopneumoniae DNA in Larynx}

The M. hyopneumoniae DNA load measured in the larynx of needle-syringe-vaccinated pigs in the VacS group was significantly $(p<0.05)$ lower at $28 \mathrm{dpv}$ than that measured for unvaccinated pigs from the UnVac group. The M. hyopneumonia DNA loads in the larynx of vaccinated pigs from the VacS, VacPulse, and VacEPIG groups were significantly $(p<0.05)$ lower at 49 and $91 \mathrm{dpv}$ than that of unvaccinated pigs in the UnVac group (Figure 4 ).

The M. hyopneuomniae DNA loads in the larynx of two needle-free injection devices at $49 \mathrm{dpv}$ (95\%CI for VacPulse -0.55 to 0.44 and for VacEPIG -0.35 to 0.35 , non-inferiority margin 0.18$)$ and $91 \mathrm{dpv}(95 \% \mathrm{CI}$ for VacPulse -0.30 to 0.41 and for VacEPIG -0.25 to 0.46 , non-inferiority margin 0.12 ) did not show non-inferiority to conventional needle-syringe injection.

\subsection{PCV2 Serology}

PCV2 antibody titers of vaccinated pigs in the VacS, VacPulse, and VacEPIG groups were significantly $(p<0.05)$ higher at 28,49 and $91 \mathrm{dpv}$ than those measured for unvaccinated pigs in the UnVac group (Figure 5). The lower bound of the $95 \% \mathrm{CI}$ on the difference in PCV2 ELISA at $49 \mathrm{dpv}$ (95\%CI for VacPulse -0.07 to 0.03 and for VacEPIG -0.09 to $0.01)$ did not exceed non-inferiority margin $(-0.10)$. This represents the outcome that shown non-inferiority, but not superiority. The PCV2 antibody titers in the VacPulse group 
at $91 \mathrm{dpv}$ showed non-inferiority to conventional needle-syringe injection (VacS) group (95\%CI -0.10 to 0.04 , non-inferiority margin -0.15$)$ (Figure 6).

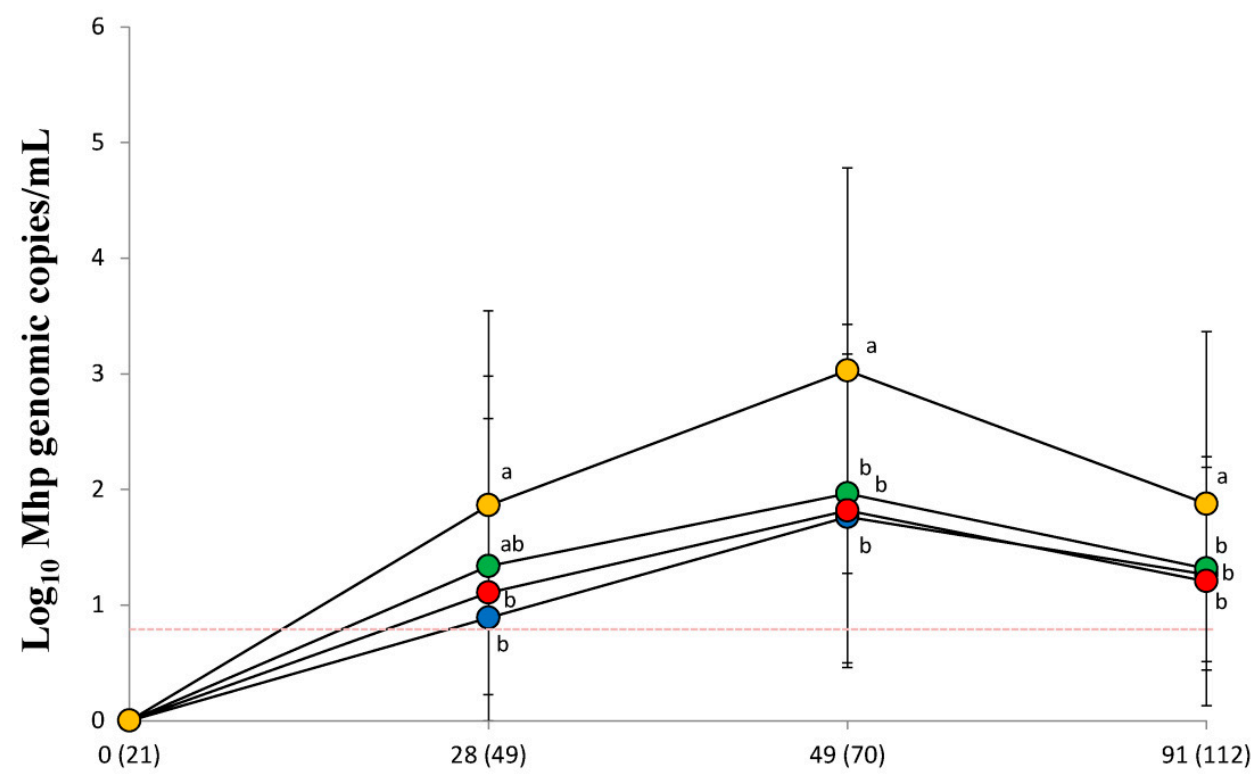

Days post-vaccination (age, days)

Figure 4. Mean values of the genomic copy number of Mycoplasma hyopneumoniae DNA in larynx from VacS $(\bullet)$, VacPulse $(\bullet)$, VacEPIG $(\bullet)$, and UnVac $(\bullet)$ groups. Variation is expressed as the standard deviation. The detection limit of the assay is 6.3 genomic copy numbers of M. hyopneumoniae (red dotted line). Different superscripts (a and b) indicate significant $(p<0.05)$ different among 4 groups.

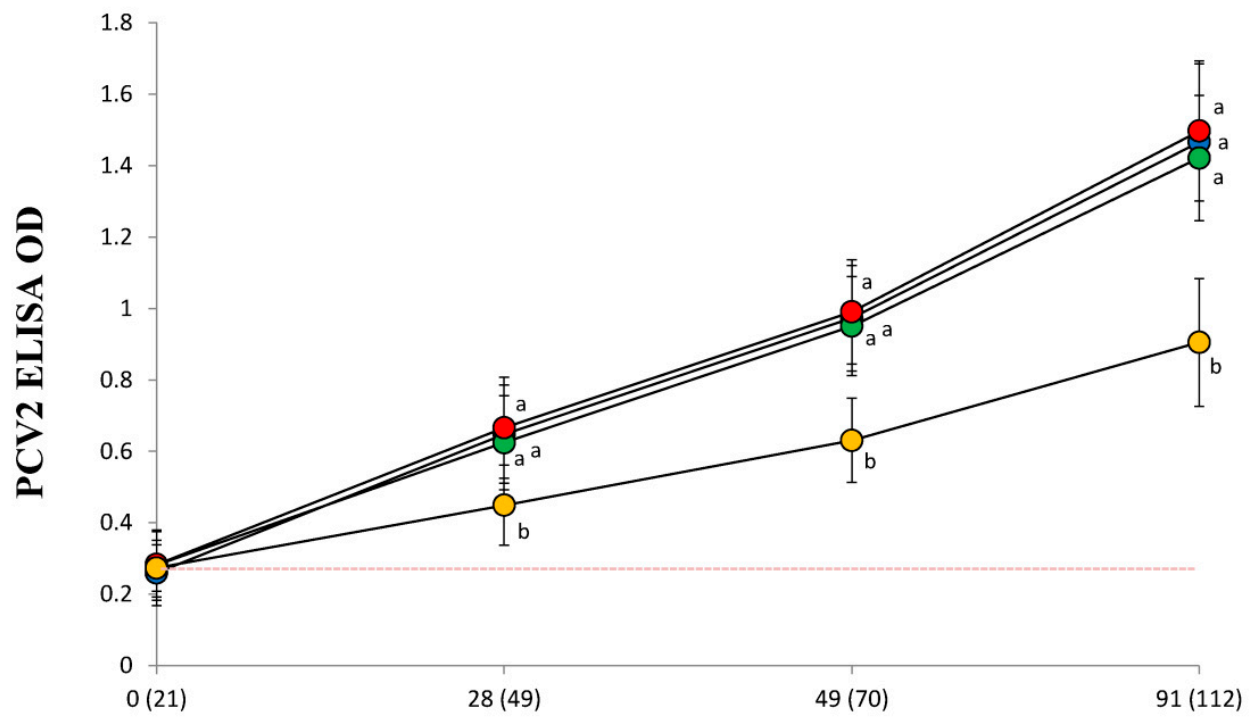

Days post-vaccination (age, days)

Figure 5. Mean values of the PCV2 antibodies by enzyme-linked immunosorbent assay (ELISA) in serum from VacS $(\bullet)$, VacPulse $(\bullet)$, VacEPIG $(\bullet)$, and UnVac $(\bullet)$ groups. Variation is expressed as the standard deviation. Serum samples are considered positive for PCV2 antibodies if the optical density (OD) is greater than 0.3 (red dotted line). Different superscripts (a and b) indicate significant $(p<0.05)$ different among 4 groups. 


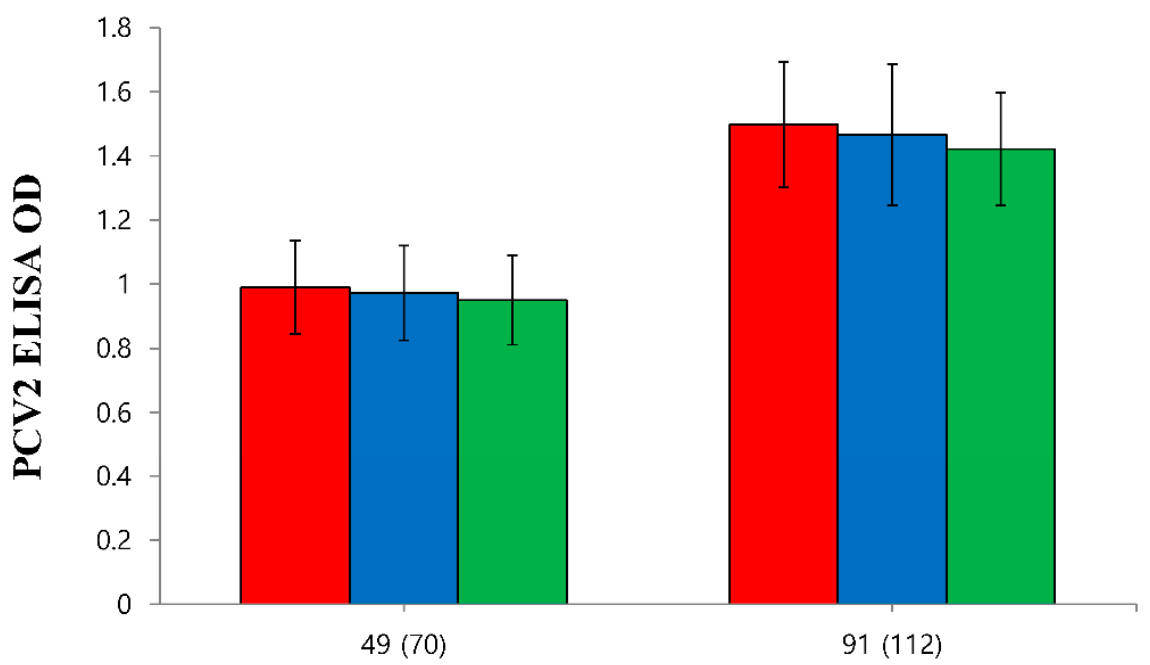

Days post-vaccination (age, days)

\begin{tabular}{lcc}
$95 \% \mathrm{Cl}$ of VacPulse & $(-0.07,0.03)$ & $(-0.10,0.04)$ \\
$95 \% \mathrm{CI}$ of VacEPIG & $(-0.09,0.01)$ & $(-0.15,0.01)$ \\
\hline Non-inferiority Margin & -0.10 & -0.15
\end{tabular}

Figure 6. The lower bound of the $95 \%$ confidence intervals (CI) on the difference in porcine circovirus type 2 (PCV2) enzyme-linked immunosorbent assay (ELISA) in the needle-free device (VacPulse, -) group at 49 days and 91 days post-vaccination (dpv) showed non-inferiority to conventional needle-syringe (VacS, $\bullet$ ) group. The lower bound of the $95 \% \mathrm{CI}$ on the difference in PCV2 ELISA in the needle-free device (VacEPIG, •) group at $49 \mathrm{dpv}$ showed non-inferiority to conventional needle-syringe $(\mathrm{VacS}, \bullet)$ group.

\subsection{Mycoplasma hyopneumoniae Serology}

M. hyopneumoniae antibody titers of vaccinated pigs in the VacS, VacPulse and VacEPIG groups were significantly $(p<0.05)$ higher at 28, 49 and $91 \mathrm{dpv}$ than those measured for unvaccinated pigs in the UnVac group (Figure 7). The lower bound of the $95 \% \mathrm{CI}$ on the difference in M. hyopneumoniae antibody titers at $49 \mathrm{dpv}(95 \% \mathrm{CI}$ for VacPulse -0.05 to 0.10 and for VacEPIG -0.04 to 0.11$)$ did not exceed non-inferiority margin $(-0.06)$. This represents the outcome that shown non-inferiority, but not superiority. The M. hyopneumoniae antibody titers in the VacPulse group at $91 \mathrm{dpv}$ showed non-inferiority against the VacS group $(95 \%$ CI -0.07 to 0.06 , non-inferiority margin -0.08) (Figure 8 ).

\subsection{Pathology}

The pigs from the vaccinated group (VacPulse, VacEPIG and VacS) had significantly $(p<0.05)$ lower macroscopic and microscopic lung lesion scores than from pigs in the UnVac group (Table 3). Compared to the VacS group, macroscopic lung lesions had an $\mathrm{OR}=0.96$ (95\%CI 0.47 to 2.00) for the VacPulse group and an OR = 1.19 (95\%CI 0.57 to 2.46) for the VacEPIG group. The VacPulse and VacEPIG groups had higher odds of microscopic lung lesions (VacPulse OR $=11.34,95 \%$ CI 0.63 to 2.89; VacEPIG OR = 1.50, 95\%CI 0.70 to 3.24) compared to the VacS group, but they were not statistically different (Table 4).

The pigs in the vaccinated groups (VacPulse, VacEPIG, and VacS) had significantly $(p<0.05)$ lower microscopic lymphoid lesion scores than pigs in the UnVac group (Table 3$)$. Compared to the VacS group, microscopic lymphoid lesions had an OR $=0.56$ (95\%CI 0.23 to 1.32) for the VacPulse group and an $\mathrm{OR}=1.03$ (95\%CI 0.28 to 1.52) for the VacEPIG group, and they were not statistically different between vaccinated groups (Table 4). 


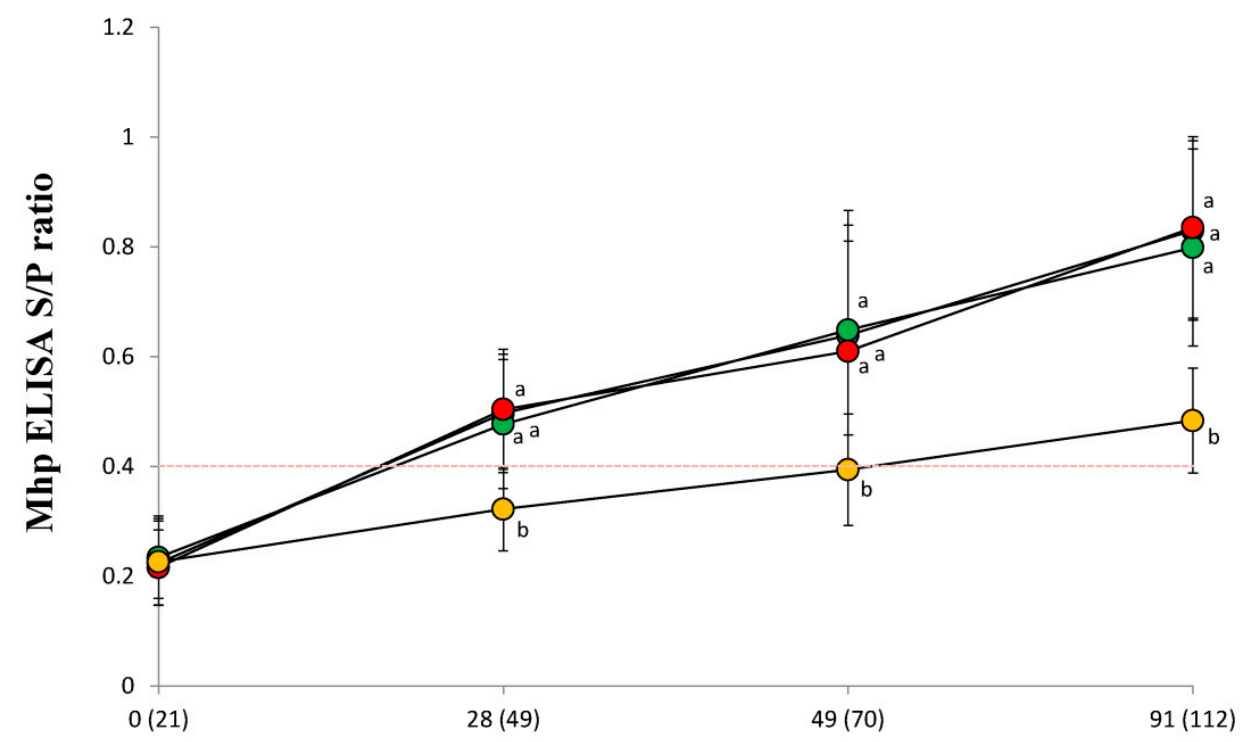

Days post-vaccination (age, days)

Figure 7. Mean values of the Mycoplasma hyopneumoniae antibodies by enzyme-linked immunosorbent assay (ELISA) in serum from VacS $(\bullet)$, VacPulse $(\bullet)$, VacEPIG $(\bullet)$, and UnVac $(\bullet)$ groups. Variation is expressed as the standard deviation. Serum samples are considered positive for M. hyopneumoniae antibodies if the sample-to-positive ( $\mathrm{S} / \mathrm{P}$ ) ratio was $\geq 0.4$ (red dotted line). Different superscripts (a and $b$ ) indicate significant $(p<0.05)$ different among 4 groups.

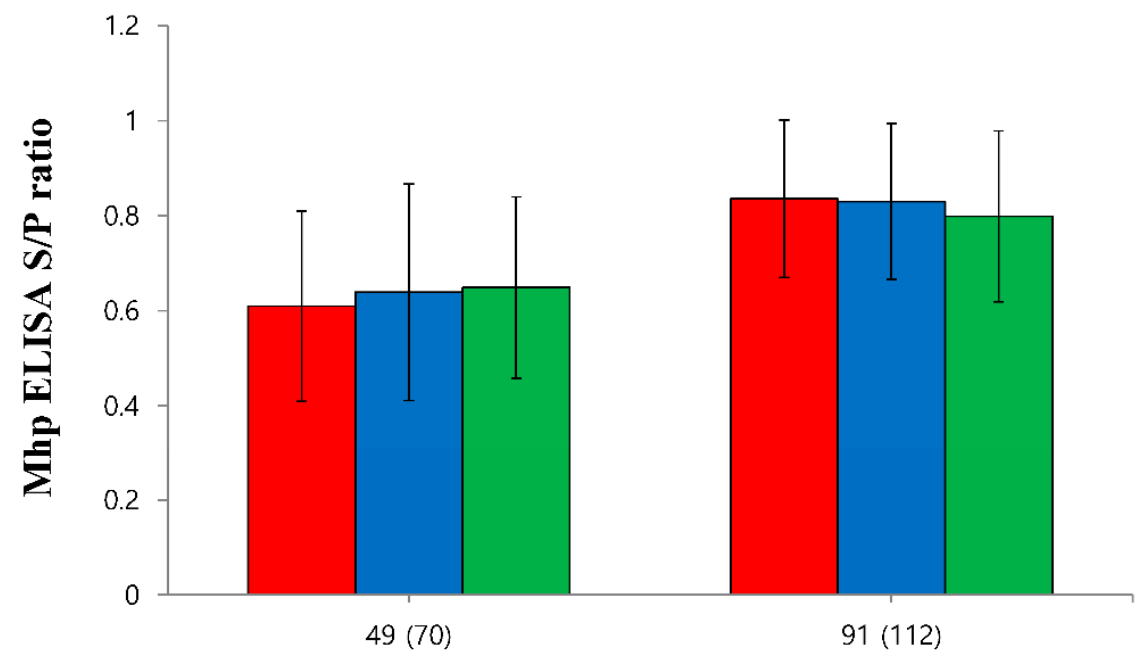

Days post-vaccination (age, days)

\begin{tabular}{lcc}
$95 \% \mathrm{Cl}$ of VacPulse & $(-0.05,0.10)$ & $(-0.07,0.06)$ \\
$95 \% \mathrm{CI}$ of VacEPIG & $(-0.04,0.11)$ & $(-0.10,0.03)$ \\
\hline Non-inferiority Margin & -0.06 & -0.08
\end{tabular}

Figure 8. The lower bound of the $95 \%$ confidence intervals (CI) on the difference in Mycoplasma hyopneumoniae enzyme-linked immunosorbent assay (ELISA) in the needle-free device (VacPulse, -) group at 49 days and 91 days post-vaccination (dpv) showed non-inferiority to conventional needle-syringe $(\mathrm{VacS}, \bullet$ ) group. The lower bound of the $95 \% \mathrm{CI}$ on the difference in M. hyopneumoniae ELISA in the needle-free device (VacEPIG, $\bullet$ ) group at $49 \mathrm{dpv}$ showed non-inferiority to conventional needle-syringe $(\mathrm{VacS}, \bullet)$ group. 
Table 3. Macroscopic and microscopic pathology (mean \pm standard deviation) of vaccinated and unvaccinated groups.

\begin{tabular}{ccccc}
\hline & \multicolumn{3}{c}{ Groups } \\
\cline { 2 - 4 } & \multicolumn{1}{c}{ VacS } & VacPulse & VacEPIG & UnVac \\
\hline $\begin{array}{l}\text { Macroscopic } \\
\text { lung lesions }\end{array}$ & $16.92 \pm 9.72^{\mathrm{a}}$ & $18.08 \pm 10.34^{\mathrm{a}}$ & $19.66 \pm 9.87^{\mathrm{a}}$ & $31.62 \pm 11.05^{\mathrm{b}}$ \\
$\begin{array}{l}\text { Microscopic } \\
\text { lung lesions }\end{array}$ & $0.73 \pm 0.62^{\mathrm{a}}$ & $0.82 \pm 0.71^{\mathrm{a}}$ & $0.92 \pm 0.68^{\mathrm{a}}$ & $2.06 \pm 0.59^{\mathrm{b}}$ \\
$\begin{array}{c}\text { Microscopic } \\
\text { lymphoid lesions }\end{array}$ & $0.56 \pm 0.57^{\mathrm{a}}$ & $0.51 \pm 0.47^{\mathrm{a}}$ & $0.60 \pm 0.52^{\mathrm{a}}$ & $1.76 \pm 0.63^{\mathrm{b}}$ \\
\hline Different superscripts $(\mathrm{a}$ and $\mathrm{b})$ indicate significant $(p<0.05)$ difference among 4 groups.
\end{tabular}

Different superscripts $(\mathrm{a}$ and $\mathrm{b})$ indicate significant $(p<0.05)$ difference among 4 groups.

Table 4. Odds ratio (OR) and 95\% confidence intervals (CI) for pathological outcome.

\begin{tabular}{ccccccc}
\hline & \multicolumn{2}{c}{$\begin{array}{c}\text { Macroscopic } \\
\text { Lung Lesions }\end{array}$} & \multicolumn{2}{c}{$\begin{array}{c}\text { Microscopic } \\
\text { Lung Lesions }\end{array}$} & \multicolumn{2}{c}{$\begin{array}{c}\text { Microscopic } \\
\text { Lymphoid Lesions }\end{array}$} \\
\cline { 2 - 7 } & OR & $\mathbf{( 9 5 \% C I )}$ & OR & $\mathbf{( 9 5 \% C I )}$ & OR & $\mathbf{( 9 5 \% C I ) ~}$ \\
\hline VacPulse & 0.96 & $(0.47-2.00)$ & 1.34 & $(0.63-2.89)$ & 0.56 & $(0.23-1.32)$ \\
VacEPIG & 1.19 & $(0.57-2.46)$ & 1.50 & $(0.70-3.24)$ & 1.03 & $(0.28-1.52)$ \\
VacS & 1.00 & (Reference) & 1.00 & (Reference) & 1.00 & (Reference) \\
\hline
\end{tabular}

\section{Discussion}

The results of this study demonstrate that the protection conferred by the administration of the trivalent vaccine with needle-free injection devices was not inferior compared to conventional needle-syringe. A common clinical feature of subclinical PCV2 infection and enzootic pneumonia is retardation of growth performance. Therefore, it is necessary to compare the growth performance for the evaluation of vaccination with needle-free injection devices and conventional needle-syringe injection. Trivalent vaccination of pigs with either needle-free injection devices or conventional needle-syringe injection improved growth performance compared to those of unvaccinated pigs. In addition, needle-free injection devices were not inferior to conventional needle-syringe injection for growth performance.

Approximately $30 \%$ and $20 \%$ of needle-free Pulse FX and needle-free EPIG injection device-vaccinated pigs had visible vaccine residue following vaccination, respectively. Residual vaccine that remains on the skin surface following vaccination with needle-free injection devices has previously been reported [20]. Adoption of needle-free vaccination may be delayed due to this unavoidable result. Comparable antibody responses against PCV2 and M. hyopneumoniae in pigs were measured in this study between two needle-free injection devices and the conventional needle-syringe injection. The needle-free injection device may allow greater penetration into the skin and greater dispersion in underlying tissue, resulting in this comparable immune response to conventional vaccination [21-23]. Data presented in this study suggests that frequent vaccine residue from needle-free injection device-vaccinated pigs may not influence the immune response. Nevertheless, administration with needle-free injection device requires a certain level of training of vaccination technique, and this may also influence the success of right administration and potentially reduce vaccine residue. Vaccine delivery with the small needle-free orifice and high pressure could potentially damage the vaccine's antigenic component through nicking or degradation, thereby altering its antigenicity or immunogenicity. An equal immune response of trivalent vaccination between needle-syringe and needle-free devices indicated that this potential degradation of vaccine antigen did not occur.

For the immunological evaluation, trivalent vaccination of pigs with either needle-free injection devices or conventional needle-syringe injection induce significantly higher levels of PCV2 and M. hyopneumoniae antibodies compared to those of unvaccinated pigs. The immune response to trivalent vaccine given with the needle-free Pulse injection device 
was non-inferior to the immune response to trivalent vaccine given with conventional needle-syringe injection. For microbiological evaluation, the trivalent vaccination of pigs with needle-free devices was able to reduce PCV2 loads in blood and M. hyopneumoniae loads in larynx at equal levels with the conventional needle-syringe administration. The reduction in PCV2 viremia and M. hyopneumoniae laryngeal shedding is clinically significant information. Trivalent vaccination with either needle-free injection devices or conventional needle-syringe injection would decrease the risk of a potential horizontal transmission to other pigs by reducing the amount of PCV2 in circulation and M. hyopneumoniae shedding within the herd. During pathological evaluation, trivalent vaccination of pigs with needlefree injection devices was able to reduce severity of mycoplasmal-induced lung lesions and PCV2-associated lymphoid lesions at equal levels compared to conventional needle-syringe methods.

Needle-free injection device systems have many benefits for pigs and labor workers, as well as for consumers. In respect to pig welfare, it reduces the pig pain and stress caused by needle injection, leading to better general health and growth capabilities [6]. Swine workers experience enhanced safety and enhance productivity from needle-free injection device systems by eliminating the risk of accidental needle stick injuries and repetitive motion injuries attributed to manually squeezing syringe and improving the vaccination process [24]. Needle-stick injuries among swine veterinarians account for the highest number of physical injuries with 580 out of 794 surveyed veterinarians (73\%) suffering needle-stick injuries [25]. In relation to food safety, needle-free injection device systems eliminate the possibility of broken needles and damage to the pig carcass [7]. This results in significant market benefits of the meat.

To the authors' knowledge, this is the first field trial in a commercial swine operation to confirm the advantage of needle-free devices to administer vaccines intramuscularly over conventional needle-syringe vaccination technique while equating immune responses between the two. Vaccine administration via needle-free injector is less stressful for pigs, they stimulate an effective immune response, and are easy and safe for swine workers to use. The tested needle free injection devices were no inferior to needle-syringe in the effectiveness of pig vaccination offering all the benefits of needle-free vaccination.

Author Contributions: Performance of the experimental trials, H.C.; vaccination, Y.A.; data analysis, J.S. and T.O.; preparation of lab analysis, H.C. and J.S.; conceptualization, C.C.; writing of the manuscript, C.C. All authors have read and agreed to the published version of the manuscript.

Funding: This research was supported by contract research funds (Grant no. 550-20190068) of the Research Institute for Veterinary Science (RIVS) from the College of Veterinary Medicine and by the BK 21 FOUR Future Veterinary Medicine Leading Education and Research Center (Grant no. A0449-20200100).

Institutional Review Board Statement: The study was conducted according to the guideline of the Seoul National Institutional Animal Care and Use, and Ethics Committee approved protocol SNU-210518-3.

Informed Consent Statement: Not applicable.

Data Availability Statement: The data present in the study are available on request from the corresponding author.

Acknowledgments: Special thanks are due to Pulse NeedleFree System and EPIG Injection System for providing the needle-free injection device for this study.

Conflicts of Interest: The authors declare no conflict of interest. 


\section{References}

1. Chae, C. A review of porcine circovirus 2-associated syndromes and diseases. Vet. J. 2005, 169, 326-336. [CrossRef] [PubMed]

2. Chae, C. Postweaning multisystemic wasting syndrome: A review of aetiology, diagnosis and pathology. Vet. J. 2004, 168, 41-49. [CrossRef]

3. Segalés, J. Porcine circovirus type 2 (PCV2) infections: Clinical signs, pathology and laboratory diagnosis. Virus Res. 2012, 164, 10-19. [CrossRef] [PubMed]

4. $\quad$ Maes, D.; Sibila, M.; Kuhnert, P.; Segalés, J.; Haesebrouck, F.; Pieters, M. Update on Mycoplasma hyopneumoniae infections in pigs: Knowledge gaps for improved disease control. Transbound. Emerg. Dis. 2018, 65, 110-124. [CrossRef] [PubMed]

5. Kim, J.; Chung, H.K.; Chae, C. Association of porcine circovirus 2 with porcine respiratory disease complex. Vet. J. 2003, 166, 251-256. [CrossRef]

6. Martinez-Miro, S.; Tecles, F.; Ramon, M.; Escribano, D.; Hemandez, F.; Madrid, J.; Orengo, J.; Martinez-Subiela, S.; Manteca, X.; Ceron, J.J. Causes, consequences and biomarkers of stress in swine: An update. BMC Vet. Res. 2016, 12, 171. [CrossRef]

7. Chase, C.C.L.; Daniels, C.S.; Garcia, R.; Milward, F.; Nation, T. Needle-free injection technology in swine: Progress toward vaccine efficacy and pork quality. J. Swine Health Prod. 2008, 16, 254-261.

8. Otake, S.; Dee, S.A.; Rossow, K.D.; Joo, H.S.; Deen, J.; Molitor, T.W.; Pijoan, C. Transmission of porcine reproductive and respiratory syndrome virus by needles. Vet. Rec. 2002, 150, 114-115. [CrossRef]

9. Baker, S.R.; Mondaca, E.; Polson, D.; Dee, S.D. Evaluation of a needle-free device to prevent hematogenous transmission of porcine reproductive and respiratory syndrome virus. J. Swine Health Prod. 2012, 20, 123-128.

10. Imeah, B.; Penz, E.; Rana, M.; Trask, C. For the Needle-less Injector Study Team Economic analysis of new workplace technology including productivity and injury: The case of needle-less injection in swine. PLoS ONE 2020, 15, e0233599.

11. Yang, S.; Oh, T.; Park, K.H.; Cho, H.; Suh, J.; Chae, C. Experimental efficacy of a trivalent vaccine containing porcine types 2a/b and Mycoplasma hyopneumoniae against PCV2d and M. hyopneumoniae challenges. Vet. Microbiol. 2021, 258, 109100. [CrossRef] [PubMed]

12. Xiao, C.T.; Halbur, P.G.; Opriessnig, T. Global molecular genetic analysis of porcine circovirus type 2 (PCV2) sequences confirms the presence of four main PCV2 genotypes and reveals a rapid increase of PCV2d. J. Gen. Virol. 2015, 96, 1830-1841. [CrossRef] [PubMed]

13. Franzo, G.; Cortey, M.; Segalés, J.; Hughes, J.; Drigo, M. Phylodynamic analysis of porcine circovirus type 2 reveals global waves of emerging genotypes and the circulation of recombinant forms. Mol. Phylogenet. Evol. 2016, 100, 269-280. [CrossRef]

14. Park, K.H.; Chae, C. The prevalence of porcine circovirus type 2e (PCV2e) in Korean slaughter pig lymph nodes when compared with other PCV2 genotypes. Transbound. Emerg. Dis. 2021, 68, 3043-3047. [CrossRef] [PubMed]

15. Um, H.; Yang, S.; Oh, T.; Park, K.; Cho, H.; Suh, J.; Min, K.-D.; Chae, C. Comparative evaluation of growth performance between bivalent and trivalent vaccines containing porcine circovirus type 2 (PCV2) and Mycoplasma hyopneumoniae in a herd with subclinical PCV2d infection and enzootic pneumonia. Vaccines 2021, 9, 450. [CrossRef] [PubMed]

16. Halbur, P.G.; Paul, P.S.; Frey, M.L.; Landgraf, J.; Eernisse, K.; Meng, X.J.; Lum, M.A.; Andrews, J.J.; Rathje, J.A. Comparison of the pathogenicity of two US porcine reproductive and respiratory syndrome virus isolates with that of the Lelystad virus. Vet. Pathol. 1995, 32, 648-660. [CrossRef]

17. Dubosson, C.R.; Conzelmann, C.; Miserez, R.; Boerlin, P.; Frey, J.; Zimmermann, W.; Häni, H.; Kuhnert, P. Development of two real-time PCR assays for the detection of Mycoplasma hyopneumoniae in clinical samples. Vet. Microbiol. 2004, 102, 55-65. [CrossRef]

18. Opriessnig, T.; Thacker, E.L.; Yu, S.; Fenaux, M.; Meng, X.-J.; Halbur, P.G. Experimental reproduction of postweaning multisystemic wasting syndrome in pigs by dual infection with Mycoplasma hyopneumoniae and porcine circovirus type 2. Vet. Pathol. 2004, 41, 624-640. [CrossRef]

19. Kim, J.; Chae, C. Expression of monocyte chemoattractant protein-1 and macrophage inflammatory protein-1 in porcine circovirus 2-induced granulomatous inflammation. J. Comp. Pathol. 2004, 131, 121-126. [CrossRef]

20. Jones, G.F.; Rapp-Gabrielson, V.; Wilke, R.; Thacker, E.L.; Thacker, B.J.; Gergen, L.; Sweeney, D.; Wasmoen, T. Intradermal vaccination for Mycoplasma hyopneumoniae. J. Swine Health Prod. 2005, 13, 19-27.

21. Bennett, C.R.; Mundell, R.D.; Monheim, L.M. Studies on tissue penetration characteristics produced by jet injection. J. Am. Dent. Assoc. 1971, 83, 625-627. [CrossRef] [PubMed]

22. Rey, M.; Undi, M.; Rodriguez-Lecompte, J.; Joseph, T.; Morrison, J.; Yitbarek, A.; Wittenberg, K.; Tremblay, R.; Corw, G.; Ominski, $\mathrm{K}$. A study of the effectiveness of a needle-free injection device compared with a needle and syringe used to vaccinate calves against bovine viral diarrhea and infectious bovine rhinotracheitis viruses. Vet. J. 2013, 198, 235-238. [CrossRef] [PubMed]

23. Giudice, E.; Campbell, D. Needle-free vaccine delivery. Adv. Drug Deliv. Rev. 2006, 58, 68-89. [CrossRef] [PubMed]

24. Trask, C.; Bath, B.; Milosavljevic, S.; Kociolek, A.M.; Predicala, B.; Penz, E.; Adebayo, O.; Whittington, L. Evaluating swine injection technologies as a workplace musculoskeletal injury intervention: A study protocol. BioMed Res. Int. 2017, 2017, 5094509. [CrossRef] [PubMed]

25. Hafer, A.L.; Langley, R.L.; Morrow, W.E.M.; Tulis, J.J. Occupational hazards reported by swine veterinarians in the United States. J. Swine Health Prod. 1996, 4, 128-141. 